\title{
Notoriété de ParticipACTION auprès des adultes canadiens : étude transversale de suivi après sept ans
}

\author{
John C. Spence, Ph. D. (1); Guy Faulkner, Ph. D. (2); Eun-Young Lee, Ph. D. (1); Tanya Berry, Ph. D. (1); \\ Christine Cameron, M. Sc. (3); Sameer Deshpande, Ph. D. (4); Amy E. Latimer-Cheung, Ph. D. (5); \\ Ryan E. Rhodes, Ph. D. (6); Mark S. Tremblay, Ph. D. (7)
}

Cet article de recherche quantitative originale a fait l'objet d'une évaluation par les pairs.

Diffuser cet article sur Twitter

\section{Résumé}

Introduction. Dans cette étude de suivi transversale, nous avons exploré la notoriété de ParticipACTION et le niveau d'activité physique chez les Canadiens après sept ans de campagnes.

Méthodologie. Une enquête à l'échelle de la population a été menée auprès de 7282 adultes entre février 2014 et mai 2015. Elle a consisté en une série de questions destinées à évaluer la notoriété de ParticipACTION dans le Sondage indicateur de l'activité physique de 2014-2015. Divers modèles de régression logistique pondérée ont été établis pour déterminer si cette notoriété était associée aux croyances à propos de l'activité physique, aux intentions et à l'activité physique pratiquée durant les loisirs.

Résultats. Environ $20 \%$ des Canadiens avaient une connaissance spontanée de ParticipACTION et le taux de notoriété assistée de ParticipACTION était de $82 \%$. L'éducation, le revenu et le fait d'avoir des enfants étaient significativement corrélés à la notoriété au sein de la population canadienne. Le rapport de cotes (RC) ajusté de la notoriété (assistée et spontanée) de ParticipACTION était plus élevé chez les personnes à haut niveau de scolarité $(\mathrm{RC}=1,57, \mathrm{IC}$ à $95 \%: 1,04$ à 2,$39 ; \mathrm{RC}=2,00, \mathrm{IC}$ à $95 \%: 1,24$ à $3,24)$, à revenu élevé $(\mathrm{RC}=3,92$, IC à $95 \%: 2,35$ à 6,$53 ; \mathrm{RC}=2,29, \mathrm{IC}$ à $95 \%: 1,44$ à 3,62 ) et ayant des enfants ( $\mathrm{RC}=1,93$, IC à $95 \%: 1,40$ à 2,66; $\mathrm{RC}=1,70$, IC à $95 \%$ : 1,26 à 2,30). La notoriété de ParticipACTION était également positivement associée aux attentes en matière de résultats et à l'auto-efficacité, et négativement associée à l'activité physique durant les loisirs.

Conclusion. Le taux de notoriété non assistée de ParticipACTION est plus élevé qu'auparavant et, par ailleurs, les Canadiens inactifs sont plus nombreux que les Canadiens actifs à connaître l'organisme. Étant donné que ParticipACTION a principalement ciblé les parents d'enfants inactifs au cours des sept dernières années, il semble que l'organisme ait partiellement atteint ses objectifs en matière de communication.

Mots-clés : médias de masse, activité physique, auto-efficacité, changement comportemental

\section{Introduction}

La vaste majorité des adultes canadiens sont physiquement inactifs et passent la majeure partie de leurs heures d'éveil en position assise $^{1}$. Pourtant, la pratique régulière d'une activité physique offre de nombreux bienfaits pour la santé, notamment la prévention des maladies chroniques comme le diabète de type 2 , les maladies cardiovasculaires, le cancer du sein et les troubles mentaux ${ }^{2}$. Ainsi, chez

\section{Points saillants}

- La notoriété de ParticipACTION auprès de la population canadienne demeure élevée.

- Ceux qui connaissent l'organisation sont plus nombreux à avoir des enfants et à être inactifs, mais aussi à avoir des croyances positives à propos de l'activité physique.

- Les initiatives de la nouvelle mouture de ParticipACTION pourraient avoir trouvé un écho auprès des adultes canadiens inactifs, par l'entremise des campagnes concernant les enfants.

- Des écarts considérables sur le plan de la notoriété continuent à être associés au niveau de scolarité et au revenu du ménage. Les prochaines campagnes de promotion de l'activité physique devraient cibler des segments précis de la population, particulièrement les personnes défavorisées.

les femmes canadiennes, l'inactivité physique est le plus important facteur de risque de mortalité toutes causes confondues (espérance de vie réduite de 3 ans), devant le tabagisme, l'alimentation malsaine et la consommation excessive d'alcool, alors qu'elle est devancée seulement par le tabagisme chez les hommes canadiens ${ }^{3}$. Par ailleurs, les variations de prévalence des comportements sains comme l'activité physique expliquent en partie l'association bien documentée entre catégorie de revenu

Rattachement des auteurs :

1. Faculté de kinésiologie, des sports et des loisirs, Université de l'Alberta, Edmonton (Alberta), Canada

2. École de kinésiologie, Université de la Colombie-Britannique, Vancouver (Colombie-Britannique), Canada

3. Institut canadien de la recherche sur la condition physique et le mode de vie, Ottawa (Ontario), Canada

4. Faculté de gestion, Université de Lethbridge, Lethbridge (Alberta), Canada

5. École de kinésiologie et des sciences de la santé, Université Queen's, Kingston (Ontario), Canada

6. École des sciences de l'exercice, de l'éducation physique et de l'éducation sur la santé, Université de Victoria, Victoria (Colombie-Britannique), Canada

7. Groupe de recherche sur les saines habitudes de vie et l'obésité, Institut de recherche du Centre hospitalier pour enfants de l'est de l'Ontario, Ottawa (Ontario), Canada

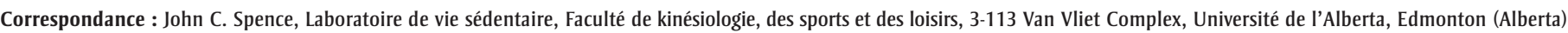
T6G 2H9; tél. : 780-492-1379; téléc. : 780-492-2364; courriel : jc.spence@ualberta.ca 
et espérance de vie aux États-Unis ${ }^{4}$. Selon une analyse économique récente, si $10 \%$ de la population canadienne inactive pouvait être amenée à être faire plus d'activité physique et à passer moins de temps assis, il en résulterait des économies d'environ 2,6 milliards de dollars d'ici 2040 pour le système de santé ${ }^{5}$. Les avantages individuels et collectifs d'une population physiquement active sont très clairs. La difficulté consiste à mettre au point des interventions et des programmes efficaces aptes à produire des changements durables à l'échelle de la population sur le plan de l'activité physique.

On a longtemps cru que les campagnes de publicité amélioraient la sensibilisation, les connaissances et les croyances à l'égard de l'activité physique ${ }^{6,7}$. Bien que diverses questions subsistent quant à la capacité des campagnes de publicité ponctuelles à accroître l'activité physique de la population $^{8,9}$, ces campagnes peuvent constituer un outil efficace pour faire la promotion de l'activité physique lorsqu'elles sont intégrées à des programmes communautaires ${ }^{10}$. Dans sa version initiale (1971-2001), ParticipACTION était un organisme de marketing social et de communication qui faisait la promotion de l'activité physique au Canada principalement par des campagnes de publicité11-13. Après une brève interruption de ses activités, l'organisme a été relancé en 2007, avec pour mandat de continuer ses campagnes et de participer au renforcement du pouvoir d'action et au partage de connaissances ${ }^{13}$. De la fin des années 1970 au début des années 2000, ParticipACTION a bénéficié d'une grande notoriété auprès de la population canadienne : son logo et ses messages étaient reconnus par $77 \%$ à $80 \%$ des Canadiens ${ }^{14}$. Bien que les activités de ParticipACTION aient été abandonnées durant six ans, l'organisme présentait auprès de la population canadienne en 2007 un taux de notoriété assistée de $82 \%$ et un taux de notoriété spontanée de $8 \%{ }^{15}$.

Le «nouveau » ParticipACTION est rapidement passé d'une approche générale visant l'ensemble de la population canadienne à une approche ciblée visant à promouvoir l'activité physique chez les enfants canadiens. Plus spécifiquement, ParticipACTION a ciblé les parents - surtout les mères - des enfants inactifs. Non seulement la majorité des enfants canadiens étaient inactifs ${ }^{16}$, mais la majorité de leurs parents croyaient qu'ils étaient suffisamment actifs physiquement $^{17,18}$. Cette perception a été corrigée principalement par des mesures d'éducation et d'information. D'abord, la campagne "Enfants inactifs » a été lancée en octobre 2007 pour accroître la sensibilisation aux effets néfastes des faibles niveaux d'activité physique sur la santé des enfants canadiens. Ensuite, la campagne "Pas si sûr » a été lancée en 2011 pour amener les mères à vérifier si leurs enfants respectaient les lignes directrices en place en matière d'activité physique ${ }^{19}$. Les deux campagnes « Recommençons à jouer » qui ont suivi offraient des solutions et des options aux parents afin de favoriser le jeu actif chez leurs enfants. Les parents qui étaient au courant de la campagne Pas si sûr avaient une meilleure connaissance des lignes directrices en matière d'activité physique, une intention plus forte d'aider leurs enfants à respecter les lignes directrices et offraient davantage de comportements de soutien envers l'activité physique de leurs enfants ${ }^{20,21}$. Ces interventions constituent dans leur ensemble de bons exemples de campagnes ciblées ayant favorisé le soutien au jeu actif et à l'activité physique par les parents ${ }^{17,18,22-24}$. Si l'on se fie aux mesures de perception dans les médias (p. ex. cote brute) et aux enquêtes-mémoire, de nombreux Canadiens ont été exposés à ces campagnes $^{13}$. Une question demeurait : celle de savoir si l'ensemble de la population canadienne était consciente des efforts de ParticipACTION.

Avant la relance de ParticipACTION en 2007, nous avons mené une enquête sur la notoriété de l'organisme auprès de la population canadienne, résultats qui ont constitué nos données de référence ${ }^{15}$. Cette enquête était fondée sur le modèle de la hiérarchie des effets ${ }^{25}$, selon lequel les campagnes de publicité influencent les comportements par une série de stades de sensibilisation et de résultats intermédiaires (p. ex. connaissances, croyances). Nous avons constaté que le degré de notoriété de ParticipACTION était associé aux croyances à propos de l'activité physique (attentes relatives aux résultats, autoefficacité, intentions) et à l'activité physique durant les loisirs et que les personnes ayant les niveaux de scolarité et de revenu les moins élevés étaient celles qui connaissaient le moins ParticipACTION.

À la lumière des nombreuses campagnes et initiatives de partage des connaissances du " nouveau » ParticipACTION depuis 2007, nous avons repris notre étude de référence pour évaluer si et comment les efforts de ParticipACTION avaient influencé les connaissances, les croyances et l'activité physique des adultes canadiens. Ainsi, cette étude transversale visait à a) évaluer la notoriété de ParticipACTION auprès de la population canadienne, b) vérifier si cette notoriété variait toujours en fonction du niveau de scolarité et du revenu et c) déterminer si elle était associée aux croyances liées à l'activité physique, aux intentions et à l'activité physique durant les loisirs, comme le suggérait le modèle de la hiérarchie des effets. Concrètement, nous avons mis à l'épreuve un modèle englobant la notoriété (spontanée et assistée) de ParticipACTION, les attentes relatives aux résultats, l'auto-efficacité, les intentions et l'activité physique durant les loisirs. Nous avons émis l'hypothèse que les individus connaissant ParticipACTION seraient plus nombreux à être physiquement actifs et à avoir des croyances positives à propos de l'activité physique.

\section{Méthodologie}

\section{Participants et procédures}

Pour évaluer la notoriété de ParticipACTION auprès de la population canadienne, nous avons mené une enquête de population tous les mois pendant 14 mois de février 2014 à mai 2015. Cette enquête consistait en une série de questions intégrées au Sondage indicateur de l'activité physique de 2014-2015, mené par l'Institut de recherche sociale de l'Université York au nom de l'Institut canadien de la recherche sur la condition physique et le mode de vie. L'échantillon à l'étude, sélectionné selon une méthode probabiliste, était proportionnel à la répartition de la population dans les provinces et territoires, avec suréchantillonnage des petites provinces. Lors du Sondage indicateur de l'activité physique de 2014-2015, les Canadiens de 18 ans et plus ont fait l'objet d'un échantillonnage transversal tous les mois dans l'ensemble des provinces et territoires. Ce processus de renouvellement continu a fourni un échantillon représentatif de la population canadienne constitué de 7282 adultes, avec un taux de réponse de $26 \%$ à $30 \%$. Nos résultats concernent l'échantillon agrégé. L'absence de réponse n’était auparavant pas associée aux caractéristiques du Sondage indicateur de l'activité physique ${ }^{26}$.

Le Sondage indicateur de l'activité physique est une enquête téléphonique à composition aléatoire qui utilise un logiciel 
d'entrevue téléphonique assistée par ordinateur. Un processus de sélection probabiliste à plusieurs étapes a été utilisé pour sélectionner un répondant âgé de 18 ans ou plus par ménage. Une fois un répondant potentiel dans un ménage sélectionné, les autres membres de ce ménage sont exclus du sondage. L'Institut de recherche sociale a administré les sondages en français ou en anglais. Les procédures de l'étude ont été approuvées par le comité d'éthique de la recherche de l'Université de l'Alberta.

\section{Mesures}

Hormis l'ajout d'une question visant à savoir si les répondants avaient des enfants, l'étude portait sur le même ensemble de variables que celui analysé en $2007^{15}$.

\section{Caractéristiques individuelles}

Les données suivantes ont été collectées auprès des participants : sexe, âge, revenu brut annuel du ménage, niveau de scolarité (études secondaires ou moins, études postsecondaires, études universitaires) et situation du ménage (avec ou sans enfants).

\section{Croyances à propos de l'activité physique} L'auto-efficacité a été mesurée à l'aide d'une question à choix de réponse sur une échelle en sept points : "Dans quelle mesure avez-vous confiance de pouvoir régulièrement faire 30 minutes ou plus d'activité physique modérée au moins trois ou quatre jours par semaine? » Les choix de réponse allaient de "Pas du tout confiant " à " Très confiant ». Les attentes relatives aux résultats ont été mesurées à l'aide de trois questions à choix de réponse sur une échelle en sept points visant à évaluer les croyances sur le fait que l'activité régulière aide à réduire le stress, à prévenir les maladies cardiaques et à poursuivre les activités de la vie quotidienne. Les choix de réponse allaient de "Pas du tout d'accord " à " Tout à fait d'accord ». Ces échelles ont ensuite été regroupées pour établir un score moyen (coefficient alpha de Cronbach $=0,72$ ). L'intention d'être physiquement actif a été mesurée à l'aide d'une seule question : "Au cours des six prochains mois, dans quelle mesure avez-vous l'intention d'être physiquement actif? ", avec un choix de réponses sur une échelle en sept points allant d'" Aucune intention » à « Intention ferme ».

\section{Notoriété de ParticipACTION}

Nous avons évalué la notoriété spontanée et la notoriété assistée de ParticipACTION.
Pour la notoriété non assistée, les participants devaient répondre à la question suivante : "Lorsque vous pensez au conditionnement physique, quel groupe ou organisation faisant la promotion de la forme physique au Canada vous vient à l'esprit? » Pour la notoriété assistée, les participants devaient indiquer s'ils connaissaient les programmes et campagnes suivants comme sources possibles d'information sur l'activité physique : les Directives canadiennes en matière d'activité physique, le Guide alimentaire canadien et ParticipACTION. Une méthode semblable avait été suivie dans les études antérieures $^{14,15,27}$.

\section{Activité physique}

L'activité physique durant les loisirs au cours des 12 derniers mois a été évaluée à l'aide d'une version adaptée du Minnesota Leisure Time Physical Activity Questionnaire $^{28}$. Les participants devaient indiquer quelles activités ils avaient pratiquées au cours de l'année (jusqu'à un maximum de 30 activités) ainsi que la fréquence et la durée de chaque activité. Le temps quotidien moyen consacré à l'activité physique durant les loisirs a été calculé selon la formule suivante : activité physique durant les loisirs (équivalent métabolique ou heures-MET $)=\Sigma\left(\mathrm{N} \_\mathrm{i} \times \mathrm{D} \_\mathrm{i} \times\right.$ MET_i/365), où $\mathrm{N}$ est le nombre de fois où l'activité $\mathrm{a}$ été pratiquée dans les 12 derniers mois, D est la durée moyenne de l'activité en heures et MET est la dépense énergétique estimée $\left(\mathrm{kJ} \times \mathrm{kg}^{-1} \times \mathrm{h}^{-1}\right)$. Les répondants consommant 3 heures-MET ou plus par jour d'activité physique durant les loisirs ${ }^{29}$, soit à peu près l'équivalent d'une marche de 60 minutes par jour, ont été classés comme actifs. Les autres répondants ont été classés comme inactifs.

\section{Analyses statistiques}

Nous avons réalisé des analyses statistiques à l'aide du logiciel SAS 9.4 (SAS Institute Inc., Cary, Caroline du Nord, États-Unis). Nous avons calculé des fréquences pour les variables relatives à la notoriété et pour les associations entre les variables sociodémographiques et l'activité physique durant les loisirs. Nous avons effectué des tests du chi carré pour examiner les différences sur le plan de la répartition du sexe, de l'âge, du revenu du ménage, du niveau de scolarité et de l'activité physique durant les loisirs, selon si les répondants avaient une connaissance spontanée ou assistée de ParticipACTION, avec un seuil de signification statistique de $p<0,05$ (objectifs a et b). Pour examiner les associations entre la notoriété (spontanée et assistée) de ParticipACTION et le statut socio-économique (revenu du ménage, niveau de scolarité), nous avons appliqué des modèles de régression logistique binaire après ajustement en fonction du sexe et de l'âge (objectif b). Pour tester le modèle de la hiérarchie des effets (objectif c), nous avons réalisé une série d'analyses de la covariance unidirectionnelles avec la procédure PROC GLM, la notoriété (assistée et spontanée) de ParticipACTION étant la variable indépendante et les attentes relatives aux résultats, l'auto-efficacité et le type de notoriété étant les variables dépendantes. Le sexe et l'âge ont été entrés à titre de covariables. Nous avons également mis au point deux modèles de régression logistique hiérarchique pour modéliser les associations entre, d'une part, l'activité physique durant les loisirs et, d'autre part, les variables " croyances » et " connaissances ", tout en apportant des ajustements destinés à tenir compte des covariables possibles. La première étape intégrait l'âge, le sexe, le niveau de scolarité, le revenu et la notoriété (assistée ou spontanée) de ParticipACTION, la deuxième intégrait les attentes relatives aux résultats et de l'auto-efficacité et la troisième, les intentions. Les tolérances et les facteurs d'inflation de la variance n'ont fait ressortir aucun problème de multicolinéarité ${ }^{30}$. Toutes les analyses ont été pondérées afin de tenir compte des plans d'échantillonnage ainsi que de la répartition par sexe et par âge de la population canadienne.

\section{Résultats}

Environ $47 \%$ des répondants ont été classés comme actifs, 82,2 \% avaient une connaissance assistée de ParticipACTION et $20,3 \%$ ont indiqué que ParticipACTION était l'organisation qui leur venait à l'esprit lorsqu'ils pensaient à la promotion de l'activité physique (tableau 1). En ce qui concerne le taux de notoriété des autres programmes et campagnes auprès des répondants, celui du Guide alimentaire canadien était de $92 \%$ et celui des Directives canadiennes en matière d'activité physique, de 37 \% (données non présentées).

La notoriété assistée comme spontanée de ParticipACTION ne variait pas de façon notable en fonction du sexe. L'âge était associé à la notoriété : les répondants les 
TABLEAU 1

Association entre certains facteurs sociodémographiques et la notoriété de ParticipACTION auprès des adultes canadiens

\begin{tabular}{|c|c|c|c|c|c|c|c|c|}
\hline & \multicolumn{8}{|c|}{ Notoriété de ParticipACTION } \\
\hline & \multicolumn{2}{|c|}{ Échantillon global } & \multicolumn{3}{|c|}{ Notoriété assistée } & \multicolumn{3}{|c|}{ Notoriété spontanée } \\
\hline & $\mathbf{N}$ & $\%$ & $\mathbf{n}$ & $\%$ & $p$ & $\mathbf{n}$ & $\%$ & $p$ \\
\hline Total & 7279 & 100 & 6526 & 82,2 & & 816 & 20,3 & \\
\hline \multicolumn{9}{|l|}{ Sexe } \\
\hline Hommes & 2920 & 48,4 & 2602 & 83,2 & & 326 & 21,6 & \\
\hline Femmes & 4359 & 51,6 & 3922 & 81,1 & & 490 & 19,0 & \\
\hline \multicolumn{9}{|l|}{ Âge (ans) } \\
\hline 18 à 24 & 284 & 25,4 & 169 & 61,3 & & 5 & 0,1 & \\
\hline 25 à 44 & 1705 & 29,0 & 1497 & 85,2 & & 228 & 26,1 & \\
\hline 45 à 64 & 3199 & 31,5 & 3030 & 93,8 & & 462 & 25,6 & \\
\hline 65 et plus & 2091 & 14,1 & 1828 & 86,9 & $<0,0001$ & 121 & 15,1 & $<0,0001$ \\
\hline \multicolumn{9}{|c|}{ Revenu brut annuel du ménage (\$) } \\
\hline Moins de 30000 & 923 & 12,0 & 762 & 68,5 & & 47 & 13,2 & \\
\hline 30000 à 59999 & 1500 & 24,1 & 1346 & 79,7 & & 134 & 13,2 & \\
\hline 60000 et plus & 3327 & 63,9 & 3125 & 88,7 & $<0,0001$ & 519 & 25,2 & $<0,0001$ \\
\hline \multicolumn{9}{|l|}{ Niveau de scolarité } \\
\hline $\begin{array}{l}\text { Études secondaires } \\
\text { ou moins }\end{array}$ & 2151 & 30,7 & 1793 & 77,4 & & 115 & 13,8 & \\
\hline Études postsecondaires & 2048 & 30,4 & 1865 & 82,4 & & 215 & 19,6 & \\
\hline Études universitaires & 3026 & 38,9 & 2828 & 85,9 & & 484 & 24,5 & $<0,01$ \\
\hline \multicolumn{9}{|l|}{ Situation du ménage } \\
\hline Sans enfants & 5911 & 80,0 & 5269 & 89,3 & & 605 & 18,3 & \\
\hline Avec enfants & 1368 & 20,0 & 1255 & 80,3 & $<0,0001$ & 211 & 26,3 & $<0,001$ \\
\hline \multicolumn{9}{|c|}{ Activité physique durant les loisirs } \\
\hline Inactif & 4040 & 53,0 & 3591 & 84,2 & & 467 & 23,4 & \\
\hline Actif & 2944 & 47,0 & 2668 & 79,8 & & 326 & 17,2 & $<0,05$ \\
\hline
\end{tabular}

Remarque : Tous les pourcentages correspondent à des données pondérées.

plus jeunes étaient moins nombreux à faire état d'une connaissance assistée que les répondants des autres groupes d'âge et, inversement, les répondants les plus âgés étaient moins nombreux que ceux des groupes de 25 à 44 ans et de 45 à 64 ans à faire état d'une connaissance spontanée. Des tendances semblables sur le plan de la notoriété ont été constatées en fonction du revenu du ménage et du niveau de scolarité : les répondants ayant les niveaux de scolarité et de revenu les moins élevés connaissaient moins ParticipACTION que les répondants ayant les niveaux plus élevés. Quel que soit le type de notoriété, les personnes ayant des enfants étaient plus nombreuses à connaître ParticipACTION. Enfin, les personnes classées comme inactives ont été plus nombreuses à déclarer une connaissance spontanée de ParticipACTION. Le niveau de scolarité, le revenu et le fait d'avoir des enfants sont restés significativement associés à la notoriété spontanée et assistée de ParticipACTION même après ajustement en fonction de l'âge et du sexe (tableau 2). Par exemple, les Canadiens gagnant plus de 60000 \$ par année étaient beaucoup plus nombreux à connaître ParticipACTION, que ce soit de façon spontanée $(\mathrm{RC}=2,29$, IC à $95 \%: 1,44$ à 3,62) ou assistée ( $\mathrm{RC}=3,92$, IC à $95 \%: 2,35$ à 6,53), que ceux gagnant moins de $30000 \$$ par année.

Pour vérifier la pertinence du modèle de la hiérarchie des effets en fonction de l'activité physique durant les loisirs, nous avons d'abord réalisé une série d'analyses de la covariance unidirectionnelles dans le but de déterminer si les attentes relatives aux résultats, l'auto-efficacité et l'intention de faire de l'activité physique variaient en fonction du degré de notoriété de ParticipACTION. Après ajustement en fonction des covariables, l'auto-efficacité $(\mathrm{F}[1,3577]=13,93, p<0,0001, \eta$-carré partiel $=0,004)$ variait significativement en fonction de la notoriété spontanée de ParticipACTION. Aucun effet semblable n'a été constaté pour les attentes relatives aux résultats ni pour les intentions. Dans le cas de la notoriété assistée de ParticipACTION, ce sont les attentes relatives aux résultats $(\mathrm{F}[1,7193]=52,44, p<0,0001, \eta$-carré partiel $=0,007$ ) qui variaient de façon significative. Aucun effet semblable n'a été constaté pour l'auto-efficacité ni les intentions. Bien que les moyennes marginales ajustées montrent que l'auto-efficacité était plus élevée chez les personnes ayant une connaissance spontanée de ParticipACTION et que les attentes les plus élevées relatives aux résultats aient été notées chez les répondants ayant une connaissance assistée de ParticipACTION (tableau 3), ces effets étaient très mineurs.

L'activité physique durant les loisirs a fait l'objet d'une régression en fonction des 
TABLEAU 2

Associations pondérées entre la notoriété (spontanée et assistée) de ParticipACTION et certaines covariables sociodémographiques ${ }^{a}$

\begin{tabular}{|c|c|c|c|c|}
\hline & \multicolumn{4}{|c|}{ Notoriété de ParticipACTION } \\
\hline & \multicolumn{2}{|c|}{ Assistée } & \multicolumn{2}{|c|}{ Spontanée } \\
\hline & RC & IC à $95 \%$ & RC & IC à $95 \%$ \\
\hline \multicolumn{5}{|c|}{ Revenu brut annuel du ménage (\$) } \\
\hline Moins de 30000 & \multicolumn{2}{|c|}{1 (référence) } & \multicolumn{2}{|c|}{1 (référence) } \\
\hline 30000 à 59999 & 1,94 & $1,07-3,52^{*}$ & 0,96 & $0,58-1,61$ \\
\hline 60000 et plus & 3,92 & $2,35-6,53^{*}$ & 2,29 & $1,44-3,62^{*}$ \\
\hline \multicolumn{5}{|l|}{ Niveau de scolarité } \\
\hline Études secondaires ou moins & \multicolumn{2}{|c|}{1 (référence) } & \multicolumn{2}{|c|}{1 (référence) } \\
\hline Études postsecondaires & 1,37 & $0,88-2,15$ & 1,51 & $0,91-2,52$ \\
\hline Études universitaires & 1,57 & $1,04-2,39^{*}$ & 2,00 & $1,24-3,24^{*}$ \\
\hline \multicolumn{5}{|l|}{ Situation du ménage } \\
\hline Sans enfants & \multicolumn{2}{|c|}{1 (référence) } & \multicolumn{2}{|c|}{1 (référence) } \\
\hline Avec enfants & 1,93 & $1,40-2,66^{*}$ & 1,70 & $1,26-2,30^{*}$ \\
\hline
\end{tabular}

Abréviations : IC, intervalle de confiance; RC, rapport de cotes.

${ }^{a}$ Ajustées en fonction du sexe et de l'âge.

${ }^{*} p<0,05$.

variables " croyances » et " connaissances » associées au modèle de la hiérarchie des effets (tableau 4), qui a révélé que l'auto-efficacité et les intentions étaient des covariables significatives quel que soit le type de notoriété. La notoriété spontanée s'est révélée être une covariable de l'activité physique durant les loisirs : si une personne connaissait ParticipACTION, la probabilité qu'elle soit physiquement active était réduite d'un facteur de 0,35.

\section{Analyse}

ParticipACTION est un organisme de marketing social et de communication qui a fait la promotion de l'activité physique durant la majorité des 45 dernières années ${ }^{13,14}$. Après une courte interruption de ses activités, il a été relancé en 2007. Nous avons constaté que la notoriété spontanée de ParticipACTION auprès de la population canadienne était d'environ $20 \%$ et que sa notoriété assistée y était de $82 \%$. Nos résultats montrent que le taux de notoriété assistée de ParticipACTION était semblable à celui de 2007, mais que le taux de notoriété spontanée, qui était de $8 \%$ en $2007^{15}$, a beaucoup augmenté entre 2007 et 2014. De manière générale, les variations individuelles sur le plan de la notoriété étaient similaires en 2007 et en 2014, excepté qu'en 2014, les répondants inactifs étaient plus nombreux que les répondants actifs à avoir une connaissance spontanée de ParticipACTION et que la proportion de femmes à avoir déclaré une connaissance spontanée de ParticipACTION était beaucoup plus élevée qu'en 2007 (6,7 \% contre $19 \%)$. De plus, en 2014, les répondants ayant des enfants étaient plus nombreux à connaître ParticipACTION que les répondants sans enfant. Aucune comparaison entre les parents et les autres adultes n’avait été faite en 2007. Comme les communications de ParticipACTION visent explicitement les parents d'enfants inactifs, cette constatation prouve que des initiatives de marketing de masse de l'activité physique réussissent à segmenter les publics en fonction de leurs caractéristiques démographiques et à offrir des communications ciblées $^{31}$. Certains auteurs avaient envisagé qu'une telle segmentation soit difficile à réaliser si le produit à vendre ou le message à transmettre avait un attrait universel, comme c'est le cas en promotion de la santé32. Nos résultats indiquent que ParticipACTION a néanmoins réussi à atteindre son public cible, en l'occurrence les parents.

Notre étude visait aussi à déterminer s'il existait des écarts sur le plan de la notoriété de ParticipACTION et, le cas échéant, si ces écarts étaient liés de façon différentielle au niveau de scolarité et au revenu. Comme c'était le cas en $2007^{15}$, la notoriété de ParticipACTION était plus importante chez les personnes à hauts niveaux de scolarité et de revenu. Ces constatations concordent avec le modèle du déficit des connaissances, selon lequel plus le niveau de scolarité d'une personne est élevé, plus celle-ci sera motivée à prêter attention aux messages en matière de santés ${ }^{33,34}$. Comme plus de $30 \%$ du gradient du niveau de scolarité lié aux comportements en matière de santé s'explique par les connaissances et par les capacités cognitives ${ }^{35}$, la réduction de ces écarts de notoriété et l'augmentation $\mathrm{du}$ savoir-faire physique devraient constituer des priorités en santé publique ${ }^{36}$. En accord avec ces constatations, la segmentation de l'information et de l'éducation du public en fonction du niveau de scolarité et de revenu constitue l'un des quatre secteurs prioritaires d'un projet de plan d'activité physique pour le Canada ${ }^{37}$.

Enfin, notre étude visait à déterminer si la notoriété de ParticipACTION était associée

TABLEAU 3

Moyennes pondérées des moindres carrés (erreurs-types) des attentes relatives aux résultats, de l'auto-efficacité et des intentions par rapport à l'activité physique selon le type de notoriété de ParticipACTION

\begin{tabular}{|c|c|c|c|c|c|c|c|c|}
\hline & \multicolumn{4}{|c|}{ Notoriété spontanée } & \multicolumn{4}{|c|}{ Notoriété assistée } \\
\hline & Non & Oui & $\boldsymbol{F}$ & ๆ-carré partiel & Non & Oui & $\boldsymbol{F}$ & $\eta$-carré partiel \\
\hline $\begin{array}{l}\text { Attentes relatives } \\
\text { aux résultats }\end{array}$ & $6,72(0,01)$ & $6,73(0,02)$ & 0,42 & 0,000 & $6,55(0,02)$ & $6,68(0,01)$ & $52,44^{* * *}$ & 0,007 \\
\hline Auto-efficacité & $5,88(0,03)$ & $5,69(0,05)$ & $13,93^{* * *}$ & 0,004 & $5,67(0,05)$ & $5,73(0,02)$ & 1,80 & 0,000 \\
\hline Intentions & $6,12(0,03)$ & $6,17(0,04)$ & 0,66 & 0,000 & $5,99(0,04)$ & $6,07(0,02)$ & 0,04 & 0,000 \\
\hline
\end{tabular}

${ }^{a}$ Ajustées en fonction du sexe et de l'âge.

${ }^{* * *} p<0,0001$. 
TABLEAU 4

Rapports de cotes ajustés et pondérés ${ }^{\mathrm{a}}$ en fonction des facteurs associés à l'activité physique durant les loisirs, selon la notoriété spontanée et assistée de ParticipACTION

\begin{tabular}{|c|c|c|c|c|c|c|c|c|c|c|c|c|}
\hline & \multicolumn{4}{|c|}{ Étape 1} & \multicolumn{4}{|c|}{ Étape 2} & \multicolumn{4}{|c|}{ Étape 3} \\
\hline & $\beta$ & Wald & RC & IC à $95 \%$ & $\beta$ & Wald & RC & IC à $95 \%$ & $\boldsymbol{\beta}$ & Wald & RC & IC à 95 \% \\
\hline \multicolumn{13}{|l|}{ Notoriété spontanée } \\
\hline Attentes relatives aux résultats & & & & & 0,22 & 1,85 & 1,29 & 0,91 à 1,72 & 0,09 & 0,27 & 1,10 & 0,77 à 1,56 \\
\hline Auto-efficacité & & & & & 0,58 & 90,10 & 1,80 & 1,59 à $2,03^{* * *}$ & 0,47 & 54,35 & 1,60 & 1,42 à $1,82^{* * *}$ \\
\hline Notoriété & 0,0079 & 0,0013 & 1,01 & 0,66 à 1,55 & $-0,04$ & 0,14 & 0,92 & 0,60 à 1,42 & $-0,04$ & 0,16 & 0,92 & 0,60 à 1,39 \\
\hline Attentes relatives aux résultats & & & & & 0,18 & 2,21 & 1,20 & 0,94 à 1,53 & 0,07 & 0,31 & 1,07 & 0,84 à 1,38 \\
\hline Auto-efficacité & & & & & 0,57 & 139,56 & 1,78 & 1,62 à $1,96^{* * * *}$ & 0,45 & 85,86 & 1,58 & 1,43 à $1,73^{* * *}$ \\
\hline Intentions & & & & & & & & & 0,47 & 55,78 & 1,60 & 1,42 à $1,81^{* * *}$ \\
\hline
\end{tabular}

Abréviations : IC, intervalle de confiance; RC, rapport de cotes.

${ }^{a}$ Ajustés en fonction du sexe, de l’âge, du niveau de scolarité, du revenu brut annuel du ménage et de la situation du ménage.

${ }^{* *} p<0,001$.

**** $p<0,0001$.

aux croyances à propos de l'activité physique, aux intentions et à l'activité physique elle-même. Les attentes relatives aux résultats (dans le cas de la notoriété assistée) et l'auto-efficacité (dans le cas de la notoriété spontanée) variaient de façon significative en fonction de la notoriété de ParticipACTION. Comme en 2007, les effets mesurés étaient peu importants, ce qui indique que des facteurs autres que la notoriété ont une incidence sur les croyances et les intentions relatives à l'activité physique. Ces constatations renforcent la validité du modèle de la hiérarchie des effets, selon lequel la notoriété est associée à des cognitions sociales positives. Cependant, selon une autre interprétation - ne pouvant être exclue en raison de la nature transversale de l'étude -, les répondants ayant une perception plus positive de l'activité physique (p. ex. auto-efficacité, attentes relatives aux résultats) seraient plus réceptifs aux messages concernant l'activité physique.

La notoriété de ParticipACTION a aussi été associée à l'activité physique durant les loisirs. Les résultats sont cependant allés à l'encontre des hypothèses émises. En effet, les personnes inactives étaient celles qui connaissaient le mieux ParticipACTION (notoriété tant spontanée qu'assistée). Cette constatation infirme l'hypothèse voulant que les personnes actives ou ayant une perception positive de l'activité physique prêtent davantage attention aux messages concernant l'activité physique ${ }^{38}$. Elle s'explique peut-être par le fait que les campagnes et les autres initiatives de la nouvelle mouture de ParticipACTION ont trouvé un écho chez les Canadiens inactifs ${ }^{39}$. Par conséquent, conformément au modèle de la hiérarchie des effets, il est possible que nous ayons fait ressortir une cascade d'effets cognitifs et comportementaux découlant des campagnes (c.-à-d. les personnes connaissant ParticipACTION auraient des croyances positives à propos de l'activité physique dans les loisirs, mais n'auraient pas encore modifié leurs comportements). De même, il est probable que les personnes alternent entre intérêt et désintérêt au fil des campagnes s'échelonnant sur plusieurs années $^{40}$. Malheureusement, la nature transversale de notre étude ne nous permet pas de déterminer si les Canadiens inactifs étaient susceptibles de modifier leurs comportements dans l'avenir. Cette modification de la notoriété pourrait aussi s'expliquer par la nature plus ciblée des communications de la nouvelle mouture de ParticipACTION, en 2007 et en 2014, en visant les parents d'enfants inactifs ${ }^{13}$. Il est donc possible que les campagnes et les messages de l'organisme aient trouvé un écho chez les adultes canadiens qui ne cherchaient pas nécessairement à modifier leur propre degré d'activité physique, mais plutôt celui de leurs enfants. Pour tenir compte de cette explication, nous avons ajusté nos régressions logistiques en fonction de la situation du ménage, mais l'association négative s'est maintenue entre l'activité physique durant les loisirs et la notoriété. Selon une autre explication, la nature affective des messages et des campagnes concernant les enfants pourrait avoir atteint et intéressé un public plus large que les parents exclusivement (p. ex. grands-parents, professeurs).

\section{Points forts et limites}

Parmi les points forts de l'étude, notons les méthodes solides d'enquête et d'échantillonnage du système de surveillance du Sondage indicateur de l'activité physique (qui fonctionne depuis longtemps), l'utilisation des mêmes mesures qu'en 2007 et l'application d'un cadre théorique pour orienter la sélection des questions et l'analyse des données. Notre étude comporte néanmoins quelques limites. La fiabilité de nos résultats pourrait être limitée par l'utilisation de mesures d'un seul élément pour évaluer l'auto-efficacité, les intentions et la notoriété, ainsi que par le faible taux de réponse des participants. Cependant, toutes ces mesures ont été utilisées sans problème dans des études antérieures ${ }^{14,15,39}$ et la mesure de l'auto-efficacité se rapporte spécifiquement au comportement, à la demande situationnelle et au temps disponible, conformément aux recommandations des experts en la matière ${ }^{41}$. De plus, comme nous l'avons mentionné précédemment, le plan transversal de l'étude ne permet pas d'analyser les causes et les effets et restreint l'application du modèle de la hiérarchie des effets. Bien que nous ayons été en mesure de tenir compte de certains facteurs individuels susceptibles d'avoir contribué aux associations observées, d'autres variables (p. ex. perception de 
l'état de santé, statut d'immigrant) pourraient avoir constitué des facteurs de confusion dans les associations entre les intentions ou l'activité physique durant les loisirs et la notoriété. Enfin, nous ne disposions d'aucune mesure de l'exposition médiatique ni du degré de notoriété des campagnes de ParticipACTION (c.-à-d. message et contenu) auprès des répondants. Ce type d'information permettrait d'éclaircir l'importance des écarts de notoriété ${ }^{42}$.

\section{Conclusion}

La notoriété de ParticipACTION demeure élevée auprès de la population canadienne. Les personnes qui connaissent l'organisme sont plus nombreuses à avoir des enfants et à être inactives, mais aussi à avoir des croyances positives à propos de l'activité physique. Les initiatives de la nouvelle mouture de ParticipACTION pourraient avoir trouvé un écho chez les adultes canadiens inactifs par l'entremise des campagnes concernant les enfants. Des écarts de notoriété considérables continuent d'être associés au niveau de scolarité et au revenu du ménage. Par conséquent, les campagnes, programmes et initiatives stratégiques en matière d'activité physique devraient cibler des segments précis de la population, particulièrement les personnes défavorisées.

\section{Remerciements}

Sept des auteurs (JCS, GF, TB, SD, ALC, RR, MST) ont siégé au groupe consultatif de recherche de ParticipACTION. Nous remercions l'Institut canadien de la recherche sur la condition physique et le mode de vie (ICRCP) de nous avoir permis d'inclure des questions sur ParticipACTION dans son Sondage indicateur de l'activité physique. Ce projet a été rendu possible par une subvention de fonctionnement des Instituts de recherche en santé du Canada (MOP123491). GF est titulaire d'une chaire en santé publique appliquée des Instituts de recherche en santé du Canada et de l'Agence de la santé publique du Canada. TB et ALC reçoivent du soutien du Programme des chaires de recherche du Canada.

\section{Conflits d'intérêts}

JS, GF, TB, SD, AER, RER et MST sont membres du Groupe consultatif de recherche de ParticipACTION.

\section{Contributions des auteurs et avis}

JCS a conçu l'étude, dirigé l'analyse des données et rédigé la première version du manuscrit. CC a dirigé la collecte de données et EYL a contribué à l'analyse et à la rédaction. GF, TB, SD, AELC, RER et MST ont contribué à la conception de l'étude, à l'analyse et à l'interprétation des données ainsi qu'à la rédaction et la révision du manuscrit. Tous les auteurs ont lu et approuvé la version finale du manuscrit.

Le contenu de l'article et les points de vue qui y sont exprimés n'engagent que les auteurs et ne correspondent donc pas nécessairement à ceux du gouvernement du Canada.

\section{Références}

1. Colley RC, Garriguet D, Janssen I, Craig CL, Clarke J, Tremblay MS. Activité physique des adultes au Canada : résultats d'accélérométrie de l'Enquête canadienne sur les mesures de la santé de 2007 à 2009. Rapports sur la santé. 2011;22(1):1-9.

2. Warburton DE, Bredin SS. Health benefits of physical activity: a systematic review of current systematic reviews. Curr Opin Cardiol. 2017;32:541-556. doi: $10.1186 / 1479-5868-7-40$.

3. Manuel DG, Perez R, Sanmartin C, et al. Measuring burden of unhealthy behaviours using a multivariable predictive approach: life expectancy lost in Canada attributable to smoking, alcohol, physical inactivity, and diet. PLoS Med. 2016;13(8):e1002082. doi: 10.1371/journal.pmed.1002082.

4. Chetty R, Stepner M, Abraham S, et al. The association between income and life expectancy in the United States, 2001-2014. JAMA. 2016;315(16);17501766. doi: 10.1001/jama.2016.4226.

5. Bounajm F, Dinh T, Thériault L. Activons-nous! Agir pour réduire l'inactivité physique et la sédentarité. Ottawa (Ont.) : Le Conference Board du Canada; 2015. En ligne à : https:// sencanada.ca/content/sen/committee /412/SOCI/Briefs/2015-06-22Activons -nousreport(ParticpACTIONAdditional info)_f.pdf
6. Cavill N, Bauman A. Changing the way people think about healthenhancing physical activity: do mass media campaigns have a role? J Sports Sci. 2004;22:771-790. doi: 10. 1080 /02640410410001712467.

7. Leavy JE, Bull FC, Rosenberg M, Bauman A. Physical activity mass media campaigns and their evaluation: a systematic review of the literature 2003-2010. Health Educ Res. 2011; 7:cyr069. doi: 10.1093/her/cyr069.

8. Abioye AI, Hajifathalian K, Danaei G. Do mass media campaigns improve physical activity? a systematic review and meta-analysis. Arch Pub Health. 2013;71(20):1-10. doi: 10.1186/0778 $-7367-71-20$.

9. Brown DR, Soares J, Epping JM, et al. Stand-alone mass media campaigns to increase physical activity: a community guide updated review. Am J Prev Med. 2012;43(5):551-561. doi: 10.1016 /j.amepre.2012.07.035.

10. Heath GW, Parra DC, Sarmiento OL, et al. Evidence-based intervention in physical activity: lessons from around the world. Lancet. 2012;380(9838):272281. doi: 10.1016/50140-6736(12) 60816-2.

11. Costas-Bradstreet C. Mobilisation, éducation et leadership : des stratégies de diffusion efficaces. Revue canadienne de santé publique. 2004; 95(suppl. 2):S27-S31.

12. Edwards P. Trente ans de marketing et de communications efficaces. Revue canadienne de santé publique. 2004; 95(suppl. 2):S6-S13.

13. Faulkner G, Yun L, Tremblay MS, Spence JC. Exploration de l'impact du " nouveau » ParticipACTION : aperçu et présentation du numéro spécial. Promotion de la santé et prévention des maladies chroniques au Canada. 2018;38(4):173-82.

14. Bauman A, Madill J, Craig CL, Salmon, A. ParticipACTION a-t-il eu les effets visés ? Revue canadienne de santé publique. 2004;95(suppl. 2): S15-S21. 
15. Spence JC, Brawley LR, Craig CL, et al. ParticipACTION: Awareness of the participACTION campaign among Canadian adults-examining the knowledge gap hypothesis and a hierarchy-of-effects model. Int J Behav Nutr Phys Act. 2009;6(85):1-9. doi: 10.1186/1479-5868-6-85.

16. Colley RC, Garriguet D, Janssen I, Craig CL, Clarke J, Tremblay MS. Activité physique des enfants et des jeunes au Canada : résultats d'accélérométrie de l'Enquête canadienne sur les mesures de la santé de 2007-2009. Rapports sur la santé. 2011;22(1):18-26.

17. Berry TR, Craig CL, Faulkner G, et al. Mothers' intentions to support children's physical activity related to attention and implicit agreement with advertisements. Int $\mathrm{J}$ Behav Med. 2014;21(1):131-138. doi: 10.1007/s12529 $-012-9279-5$.

18. Faulkner G, Solomon V, Berry T, et al. Examining the potential disconnect between parents' perceptions and reality regarding the physical activity levels of their children. JARC. 2014;5(1): 1-29. En ligne à : http://digitalcommons .library.tmc.edu/cgi/viewcontent.cgi ? article $=1156 \&$ context $=$ childrenatrisk

19. Tremblay MS, Warburton DE, Janssen I, et al. New Canadian physical activity guidelines. Appl Physiol Nutr Metab. 2011;36(1):36-46. doi: 10.1139 /H11-009.

20. Gainforth HL, Jarvis JW, Berry TR, et al. Evaluating the ParticipACTION "Think Again" Campaign. Health Educ Behav. 2015;43(4):434-441. doi: $10.1177 / 1090198115604614$.

21. Jarvis JW, Rhodes RE, Deshpande S, et al. Investigating the role of brand equity in predicting the relationship between message exposure and parental support for their child's physical activity. Soc Mark Q. 2014;20(2): 103-115. En ligne à : http://journals .sagepub.com/doi/abs/10.1177/15245 00414528183.

22. Rhodes RE, Berry T, Craig CL, et al. Understanding parental support of child physical activity behavior. Am J Health Behav. 2013;37(4):469-77. doi: 10.5993/AJHB.37.4.5.
23. Rhodes RE, Spence JC, Berry T, et al. Predicting changes across 12 months in three types of parental support behaviors and mothers' perceptions of child physical activity. Ann Behav Med. 2015; 49(6):853-864. doi: 10.1007 /s12160-015-9721-4.

24. Rhodes RE, Spence JC, Berry T, et al. Understanding action control of parent support behavior for child physical activity. Health Psychol. 2016;35(2): 131-140. doi: 10.1037/hea0000233.

25. McGuire WJ. Public communication as a strategy for inducing health-promoting behavioral change. Prev Med. 1984;13:299-319.

26. Craig CL, Tudor-Locke C, Bauman A. Twelve-month impact of Canada on the Move: A population-wide campaign to promote pedometer use and walking. Health Educ Res. 2007;2:406413. doi: 10:1093/her/cyl093.

27. Craig CL, Cragg SE, Tudor-Locke C, Bauman A. Proximal impact of Canada on the Move: The relationship of campaign awareness to pedometer ownership and use. Can J Pub Health. 2006; 97(1 Suppl):S21-27.

28. Taylor HL, Jacobs DR, Schucker B, Knudsen J, Leon AS, Debacker G. A questionnaire for the assessment of leisure time physical activities. J Chronic Dis. 1978;31(12):741-755.

29. Craig CL, Russell SJ, Cameron C, Bauman A. Twenty-year trends in physical activity among Canadian adults. Can J Pub Health. 2004:95(1); 59-63.

30. Craney TA, Surles JG. Modeldependent variance inflation factor cutoff values. Qual Eng. 2002;14(3): 391-403. doi: 10.1081/QEN-120001878.

31. Latimer AE, Brawley LR, Bassett RL. A systematic review of three approaches for constructing physical activity messages: What messages work and what improvements are needed? Int J Behav Nutr Phys Act. 2010 May 11;7(36): 1-17. doi: 10.1186/1479-5868-7-36.

32. Slater MD, Flora JA. Health lifestyles: Audience segmentation analysis for public health interventions. Health Educ Behav. 1991;18:221-33.
33. Gaziano C. The knowledge gap: an analytical review of media effects. Communication Research. 1983;10(4): 447-486.

34. Kwak N. Revisiting the knowledge gap hypothesis: education, motivation, and media use. Comm Res. 1999;26: 385-413. doi: 10.1177/009365099026 004002 .

35. Cutler DM, Lleras-Muney A. Understanding differences in health behaviors by education. J Health Econ. 2010; 29:1-28. doi: 10.1016/j.jhealeco.2009 .10.003.

36. Piirtola M, Kaprio J, Kujala UM, et al. Association between education and future leisure-time physical inactivity: a study of Finnish twins over a 35-year follow-up. BMC Pub Health. 2016; 16(720):1-11. doi: 10.1186/s12889-016 $-3410-5$.

37. Spence JC, Faulkner G, Bradstreet CC, Duggan M, Tremblay MS. Active Canada 20/20: A physical activity plan for Canada. Can J Pub Health. 2016; 106(8):E470-473. En ligne à : https:// journal.cpha.ca/index.php/cjph /article/viewFile/5041/3278

38. Berry TR. Who's even interested in the exercise message? Attentional bias for exercise and sedentary-lifestyle related words. J Sport Exer Psych. 2006;28(1): 4-17. doi: 10.1123/jsep.28.1.4.

39. Craig CL, Bauman A, Latimer-Cheung $A$, et al. An evaluation of the "My ParticipACTION" campaign to increase self-efficacy for being more physically active. J Health Commun. 2015;20(9): 995-1003. doi: 10.1080/10810730.2015 .1012240 .

40. Leavy JE, Rosenberg M, Bull F, Bauman, A. Who do we reach? Campaign evaluation of Find Thirty every day ${ }^{\circledR}$ using awareness profiles in a Western Australian cohort. J Health Commun. 2014;19:853-869. doi: 10.1080 /10810730.2013.837560.

41. Maibach E, Murphy DA. Self-efficacy in health promotion research and practice: conceptualization and measurement. Health Educ Res. 1995;10: 37-50. doi: 10.1093/her/10.1.37. 
42. Kang Y. Knowledge gap effect in health campaign evaluations. 59 $9^{\text {th }}$ Annual International Communications Association Conference, May 21-25; Chicago (Ill)/New York : ICA Press; 2009. En ligne à : http://www.allacademic.com /meta/p11702_index.html 